\title{
Memex Is Not Enough
}

\author{
Richard Mark Soley \\ Object Management Group, Inc. \\ Needham, Massachusetts
}

Turning his thinking finally from the application of science to military needs, in 1946 the great scientist and research organizer Dr. Vannevar Bush of the United States' wartime Office of Scientific Research and Development wrote a seminal paper entitled As We May Think. In it, he challenged us to discover ways to encode, link and in general make more accessible the huge store of human knowledge developed to that date.

In the last decade, the emergence of the World Wide Web has been held up as the embodiment of that half-century-old vision. While this is a reasonable claim, even if true it is simply not enough. Human beings, and more importantly our silicon-based information processors, do not simply encode, store and index static data; they create new data every nanosecond. Links between static data, stale the moment they are created, are simply not enough; the real challenge is to link the information processing power we have directly.

At the core of this effort must be sufficient consensus for interfaces not only to store and link data, but to forward and link computational power. This effort has informally been underway for decades, with thousands of "stovepiped" system thrown together without any planning. We must renew our efforts to agree standards for linking information assets, for mundane (but economically critical) "business to consumer" as well as "business to business" supply/service-chain integration, and for more interesting problems as well.

Typically such standardization efforts have centered on the lowest levels of the interoperability protocol stack, including the physical cable or the bytetransfer level. Even newer approaches based on document type extensions (e.g., XML) have focussed only on data movement. Unfortunately, it is the much more difficult piece of the puzzle that is required for true processing power synchronization: transactional, persistent, secure application interfaces (at the lower level) and market-specific interface definitions (at the higher level) are absolutely critical.

Memex - the availability of linked, indexed data - is a great starting point for integrating the sum total of human knowledge, as Bush saw a half century ago. By simply implementing this vision we abandon it; instead we should focus on providing true application-level integration across worldwide networks so that knowledge may be shared the moment it is discovered. 